\title{
Quantitative susceptibility mapping of carotid arterial tissue ex vivo: Assessing sensitivity to vessel microstructural composition
}

\author{
Alan J. Stone ${ }^{1,2}$ | Brooke Tornifoglio ${ }^{1,2}$ iD \\ Christian Kerskens $^{4}$ | Caitríona Lally ${ }^{1,2,5}$ \\ | Robert D. Johnston ${ }^{1,2}$ \\ Karin Shmueli ${ }^{3}$ \\ ${ }^{1}$ Trinity Centre for Biomedical Engineering, Trinity Biomedical Sciences Institute, Trinity College Dublin, Dublin, Ireland \\ ${ }^{2}$ Department of Mechanical, Manufacturing and Biomedical Engineering, School of Engineering, Trinity College Dublin, Dublin, Ireland \\ ${ }^{3}$ Department of Medical Physics and Biomedical Engineering, University College London, London, United Kingdom \\ ${ }^{4}$ Trinity College Institute of Neuroscience, Trinity College Dublin, Dublin, Ireland \\ ${ }^{5}$ Advanced Materials and Bioengineering Research Centre (AMBER), Royal College of Surgeons in Ireland and Trinity College Dublin, Dublin, Ireland
}

\section{Correspondence}

Caitríona Lally, Department of Mechanical,

Manufacturing and Biomedical

Engineering, School of Engineering, Trinity

College Dublin, Dublin, Ireland.

Email: lallyca@tcd.ie

Funding information

This research was funded by the European

Research Council (ERC) under the

European Union's Horizon 2020 research

and innovation programme, grant 637674

KS is funded by ERC Consolidator Grant

DiSCo MRI SFN 770939.
Purpose: To characterize microstructural contributions to the magnetic susceptibility of carotid arteries.

Method: Arterial vessels were scanned using high-resolution quantitative susceptibility mapping (QSM) at 7 Tesla. Models of vessel degradation were generated using ex vivo porcine carotid arteries that were subjected to several different enzymatic digestion treatments that selectively removed microstructural components (smooth muscle cells, collagen, and elastin). Magnetic susceptibilities measured in these tissue models were compared to those in untreated (native) porcine arteries. Magnetic susceptibility measured in native porcine carotid arteries was further compared to the susceptibility of cadaveric human carotid arteries to investigate their similarity.

Results: The magnetic susceptibility of native porcine vessels was diamagnetic $\left(\chi_{\text {native }}=-0.1820 \mathrm{ppm}\right)$, with higher susceptibilities in all models of vessel degradation $\left(\chi_{\text {elastin-degraded }}=-0.0163 \mathrm{ppm} ; \chi_{\text {collagen-degraded }}=-0.1158 \mathrm{ppm} ; \chi_{\text {decellularized }}=\right.$ $-0.1379 \mathrm{ppm} ; \chi_{\text {fixed native }}=-0.2199 \mathrm{ppm}$ ). Magnetic susceptibility was significantly higher in collagen-degraded compared to native porcine vessels (Tukey-Kramer, $P<.01)$ and between elastin-degraded and all other models (including native, Tukey-Kramer, $P<.001)$. The susceptibility of fixed healthy human arterial tissue was diamagnetic, and no significant difference was found between fixed human and fixed porcine arterial tissue susceptibilities (analysis of variance, $P>.05$ ).

Conclusions: Magnetic susceptibility measured using QSM is sensitive to the microstructural composition of arterial vessels-most notably to collagen. The similarity of human and porcine arterial tissue susceptibility values provides a solid basis for translational studies. Because vessel microstructure becomes disrupted during the onset and progression of carotid atherosclerosis, QSM has the potential to provide a sensitive and specific marker of vessel disease. 
K E Y W O R D S

arterial microstructure, atherosclerosis, carotid artery, plaque, quantitative susceptibility mapping (QSM)

\section{1 | INTRODUCTION}

\subsection{Carotid atherosclerosis and stroke}

Atherosclerosis is a widespread form of cardiovascular disease that causes the formation of plaque inside arterial vessels. Plaque formation is caused by a buildup of fat, cholesterol, calcium, fibrous tissue, and other substances that disrupt the microstructural composition of the vessel, causing narrowing of the luminal space and stiffening of the vessel. Carotid arteries are particularly prone to plaque formation, and stenosis or rupture here can have catastrophic consequences because these vessels carry the main supply of blood to the brain. An estimated $20 \%$ of ischemic stroke is due to rupture of carotid plaques, with the majority of ischemic stroke caused by stenosis. ${ }^{1,2}$ Carotid artery disease is also responsible for nearly $50 \%$ of transient ischemic attacks and linked to an increased risk of heart attack. ${ }^{3}$ Early identification and treatment is of utmost importance to avoid stroke-related disability and death caused by carotid plaque. The current indicator for surgical intervention is to assess the degree of stenosis caused by atherosclerotic plaque in the vessel. Although the patency of the blood vessel can be identified using standard diagnostic imaging techniques (MRI, CT, and ultrasound), it has been shown that improved specificity for stroke prediction can be gained from identifying plaque features, such as intraplaque hemorrhage, that are associated with increased stroke risk. ${ }^{4,5}$

\section{2 $\mid$ QSM}

Quantitative susceptibility mapping (QSM) is an MRI method capable of spatially mapping the magnetic susceptibility of biological tissue. ${ }^{6-8}$ Recently, a number of studies have applied QSM to carotid plaques and demonstrated marked improvement in intraplaque hemorrhage and calcification depiction compared to conventional multi-contrast MRI $^{9-13}$ based on the inherent differences between the magnetic susceptibilities of these structures: calcifications are diamagnetic, whereas hemorrhages are paramagnetic. ${ }^{14}$

\subsection{Arterial microstructure and QSM}

Although QSM has proven promising for evaluating carotid plaques, specific disease-driven sources of susceptibility changes have yet to be investigated in arterial vessels and plaques. Arterial tissue is predominantly composed of smooth muscle cells, elastin, and collagen that are helically arranged to form the vessel microstructure. ${ }^{15}$ The quantity, quality, and organization of this microstructure is finely tuned to maintain proper physiological function in healthy blood vessels. ${ }^{16}$ Furthermore, arterial microstructure has been shown to become disrupted during the onset and progression of atherosclerosis, and this changing microstructural arrangement may be indicative of mechanical stability, and therefore rupture risk, of advanced plaques. ${ }^{17}$ Because the magnetic susceptibility of a tissue is governed by its molecular makeup, QSM can provide insight into the microstructural composition of biological tissue. To date, QSM has been demonstrated to be sensitive to various tissue microstructural components such as myelin in brain, ${ }^{18,19}$ collagen in liver ${ }^{20,21}$ and articular cartilage, ${ }^{22-24}$ tubules in kidney, ${ }^{25}$ and myofibers in heart. ${ }^{26}$ As such, investigation of the sensitivity of QSM to arterial microstructure is warranted to determine if this is a source of susceptibility contrast that may provide an important biomarker of disease onset and progression in arteries.

\subsection{Aim and summary}

In this study, we hypothesize that the magnetic susceptibility of arterial vessels is sensitive to changes in the vessel wall microstructure. To test this, a high-resolution QSM protocol was applied to excised porcine carotid arteries subjected to a range of enzymatic digestion treatments. ${ }^{27}$ To relate the magnetic susceptibility of porcine tissue to human arterial tissue to accelerate potential clinical translation, the same QSM protocol was applied in ex vivo human carotid arteries. In both porcine and human vessels, detailed histological analysis was used to understand the underlying tissue microstructural composition and interpret the magnetic susceptibility of arterial vessels measured using QSM.

\section{2 | METHODS}

\section{1 | Sample preparation}

\subsection{1 | Porcine carotid artery (microstructural) models}

Carotid arteries were harvested from 6-month-old, large white pigs within $3 \mathrm{~h}$ of slaughter. All vessels were cleaned 
of connective tissue and cryopreserved using a protocol to preserve the integrity of tissue microstructure during freezing. ${ }^{28,29}$ In preparation for imaging, vessels were thawed at $37^{\circ} \mathrm{C}$ and rinsed in phosphate-buffered saline (PBS) (P5493, Sigma Aldrich, Wicklow, Ireland).

To investigate the sensitivity of QSM to arterial tissue microstructure, 4 different vessel models were developed with distinct microstructural compositions using porcine carotid arteries. All vessels were imaged directly after treatment and before fixation. The native vessel model refers to porcine carotid arteries that were not subjected to any treatment and acted as a control tissue for comparison with the following models. Details of the enzymatic digestion treatments used to achieve collagen-degraded, decellularized, ${ }^{30}$ and elastindegraded models are provided in the Supporting Information (Data S1).

\subsubsection{Human carotid artery}

Carotid arteries (including the carotid bifurcation and proximal sections of the common, internal, and external carotid artery) were excised from 5 embalmed cadavers. All cadaver tissue was sanctioned for research use by The Royal College of Surgeons in Ireland Research Ethics Committee and The Royal College of Surgeons in Ireland Museum Committee. One artery was obtained from each subject. The subjects ( 3 females and 2 males) ranged from 70 to 103 years in age (mean $81.6 \pm 12.7$ years). Cardiovascular disease was not implicated as the cause of death in any subjects. Vessels were cleaned of connective tissue and stored in PBS. To facilitate comparison of susceptibility values between human and porcine arteries, fixed native porcine carotid arteries were produced by immersing native porcine vessels in 4\% formalin (HT501128, Sigma) for 7 days at $4^{\circ} \mathrm{C}$. Prior to MR imaging, all samples were washed and placed in fresh PBS.

\section{2 | MR imaging}

\subsection{1 | Vessel positioning}

All vessels were positioned using 3D-printed holders composed of polylactic acid and placed in 50-mL falcon tubes (Supporting Information Figure S1) in which samples were immersed in fresh PBS prior to imaging at room temperature. PBS was chosen as the "embedding" material because it has previously been identified as providing a stable experimental setup facilitating good image quality for QSM of postmortem brain specimens. ${ }^{31}$ For the porcine vessels (native, decellularized, collagen-degraded, elastin-degraded, and fixed native), 6 vessels $(\mathrm{n}=6)$ of each model were produced, and holders were designed to secure 6 vessels in a single $50-\mathrm{mL}$ falcon tube. For human vessels, holders were designed to hold a single specimen.

\subsubsection{Image acquisition}

A small-bore $(30 \mathrm{~cm})$ horizontal 7 Tesla Bruker BioSpec 70/30 USR system (Bruker, Ettlingen, Germany) equipped with a receive-only 8-channel surface array coil, birdcage design transmit coil, shielded gradients (maximum strength $770 \mathrm{mT} / \mathrm{m}$ ), and Paravision 6 software (Bruker, Ettlingen, Germany) was used for all imaging. For each session, a test tube containing the vessels of interest was placed securely in the cradle of the 8-channel surface array coil. A total of 10 scan sessions were performed ( 5 sessions for porcine vessels, 5 sessions for human vessels).

For QSM, data were acquired using a 3D multi-echo gradient echo (ME-GRE) sequence with the following parameters: TEs $=5,13.1,21.2$, and $29.3 \mathrm{~ms}$ with monopolar readout gradients, $\mathrm{TR}=150 \mathrm{~ms}$, flip angle $=30^{\circ}$, bandwidth $=$ $34722 \mathrm{~Hz}$, and averages $=2$. The readout direction was oriented along the long axis of the tube. An isotropic voxel resolution of $0.117 \times 0.117 \times 0.117 \mathrm{~mm}^{3}$ was achieved using a field of view (FOV) of $30 \mathrm{~mm} \times 30 \mathrm{~mm} \times 30 \mathrm{~mm}$ and a $256 \times$ $256 \times 256$ matrix size. Total scan time for this sequence was 5 h 27 min.

\section{3 | Image processing}

For all samples, the multi-channel ME-GRE data were coilcombined. Coil-combined magnitude images were calculated using the root mean square (RMS) of the channels, ${ }^{32}$ and coil-combined phase images were produced using the phase difference approach. ${ }^{33}$

\subsection{1 $\quad$ QSM pipeline in porcine vessels}

The QSM and $\mathrm{R}_{2}^{*}$ calculation is summarized in Figure 1 . $\mathrm{R}_{2}^{*}$ maps were calculated using the Auto-Regression on Linear Operations algorithm ${ }^{34}$ applied to the coilcombined ME-GRE magnitude data. To aid masking, an echo-combined magnitude image was calculated using the RMS of all echoes. For QSM, an initial mask was created by thresholding the echo-combined magnitude image with the threshold set to include all vessels and PBS but to exclude the 3D-printed vessel holder and air outside the tube. The mask was manually refined to exclude air bubbles using the echo-combined magnitude and $\mathrm{R}_{2}^{*}$ map as references. Nonlinear field fitting ${ }^{35,36}$ was used to estimate field maps from the complex ME-GRE data, and remaining 


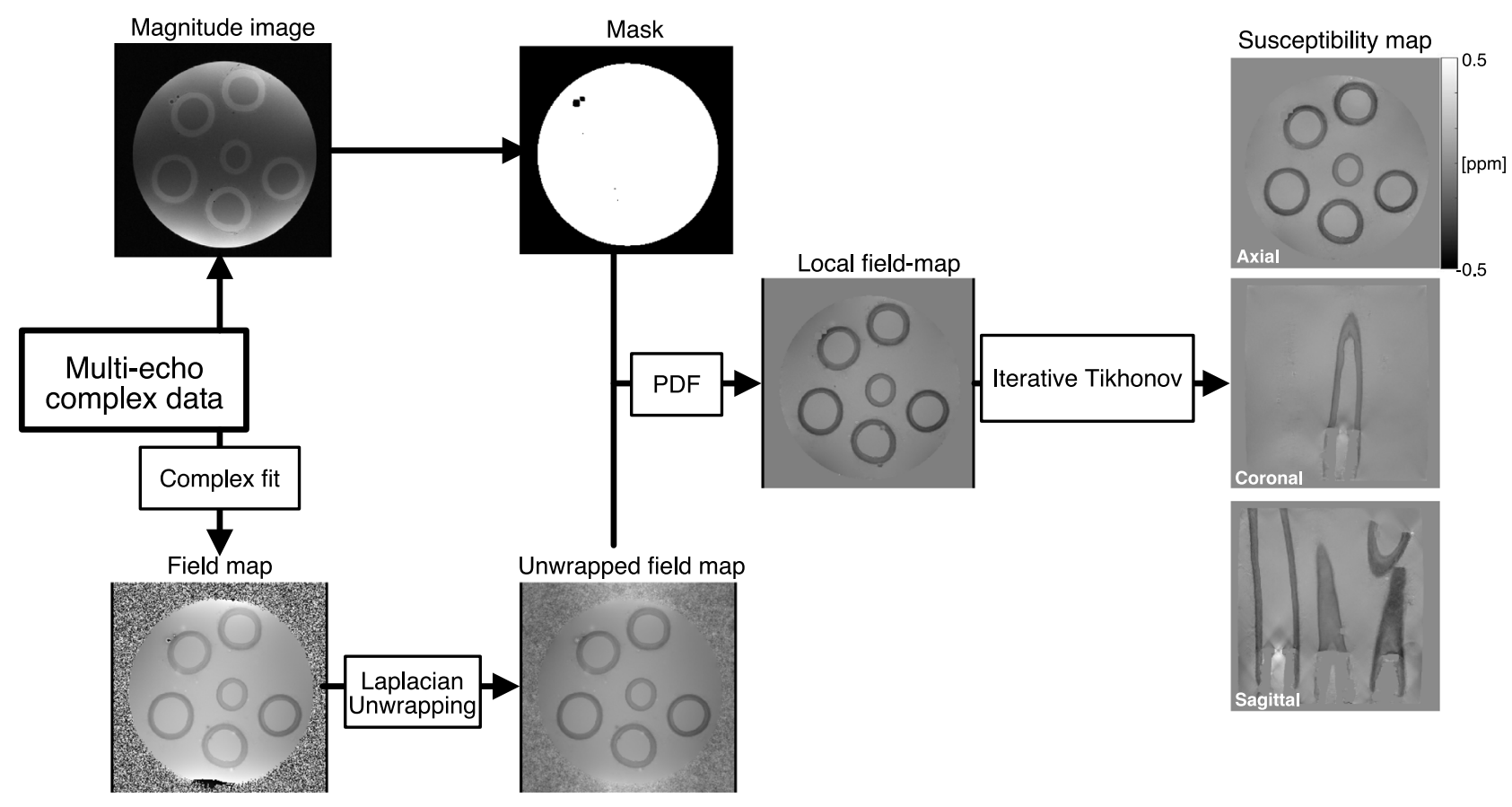

F I G URE 1 Outline of QSM pipeline for porcine carotid artery tissue models

wraps were removed using Laplacian phase unwrapping. ${ }^{37}$ This approach (nonlinear field fitting followed by Laplacian phase unwrapping) provided a computationally efficient and robust approach to calculating unwrapped total field maps and has previously been applied to investigate tissue susceptibility in ex vivo articular cartilage. ${ }^{22}$ Alternative approaches, such as linear field fitting, require prior unwrapping of individual echoes, which would lead to long computation times, particularly for the large matrix sizes acquired here $(256 \times 256 \times 256)$, whereas the approach used in this study requires unwrapping of only a single image. The local field map was calculated using the projection onto dipole fields method and the unwrapped field map and magnitude mask as input. ${ }^{38,39}$ The projection onto dipole fields method was chosen because it has been shown to perform well in comparison to alternative methods. ${ }^{40}$ No additional Spherical Mean Value filtering was applied as is the default in the morphology enabled dipole inversion (MEDI) toolbox. A susceptibility map was calculated from the local field map using an iterative Tikhonov method, ${ }^{7,41,42}$ with correction for susceptibility underestimation. ${ }^{43}$ The same regularization parameter $(\alpha)$ was used for all samples and was chosen by performing L-curve optimization ${ }^{44}$ in all 5 porcine datasets and calculating the mean of the individually optimized parameters. Alternative susceptibility calculation methods (truncated $\mathrm{k}$-space division, ${ }^{45}$ direct Tikhonov, ${ }^{7,41,46}$ and morphology enabled dipole inversion ${ }^{47}$ ) were tested and found not to influence the trends and final conclusions reported for porcine vessels (see Supporting Information Figures S9 and S10).

\subsubsection{QSM pipeline in human vessels}

All 5 human vessels scanned in this study had regions of advanced atherosclerotic disease close to the bifurcation (see Figure 7 yellow arrows). These heavily diseased regions contained structures with little or no signal that caused significant streaking in the final susceptibility maps likely attributable to the presence of calcification or hemorrhage. To investigate the impact of these low signal-to-noise (SNR) regions on the susceptibility measurements made in regions of the common carotid unaffected by disease/plaque, 2 different masking procedures were compared for QSM (Figure 2).

\section{Comparison of masking procedures for human vessels}

First, a tube mask was generated for each human vessel that included heavily diseased low SNR regions. This mask was generated by thresholding the echo-combined magnitude image to exclude the 3D-printed vessel holder and air outside the tube and include all other contents of the tube, that is, PBS and the whole vessel (including diseased regions). No manual refinement step was implemented here due to the difficulty in distinguishing diseased regions from air bubbles.

Second, a noise mask was generated for each human vessel that excluded heavily diseased low SNR regions. High noise regions in areas of advanced atherosclerotic disease were identified by thresholding the inverse noise map (calculated during nonlinear field fitting ${ }^{36}$ using noise propagation ${ }^{46}$ ) at one-third of the mean voxel value contained within the tube mask. To generate the final noise mask, the regions of high noise identified by the inverse noise map were removed from the tube mask (see Figures 2 and 8). 


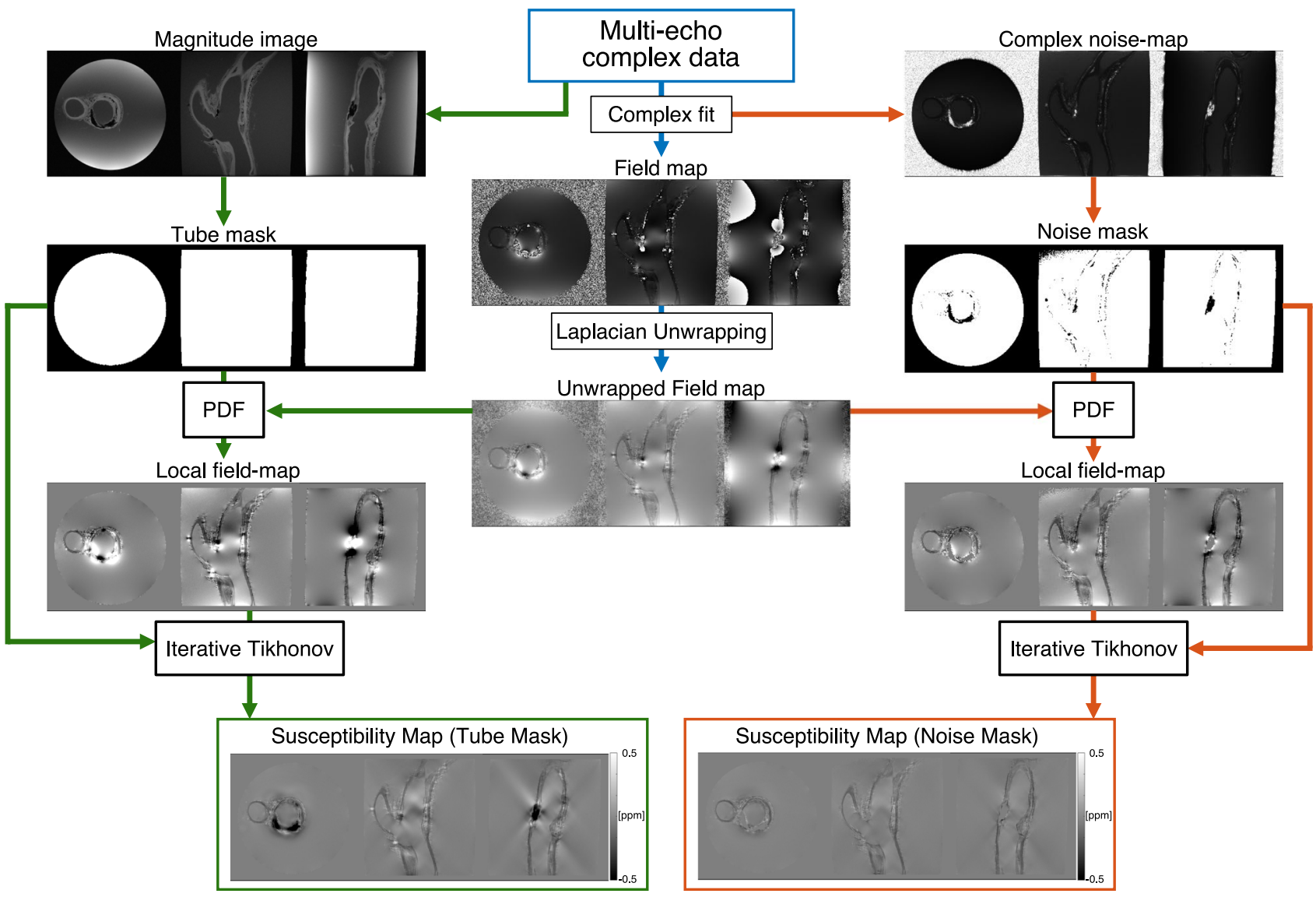

F I G URE 2 Outline of QSM pipeline for human carotid specimens. Two different masks were compared to investigate the impact of low SNR, diseased regions on the susceptibility measured in nearby healthy regions of the common carotid artery. The noise mask pipeline (orange) excluded regions of low SNR from the background field removal and susceptibility calculation steps using a mask that was calculated by thresholding the inverse of the residual noise map produced from the nonlinear fit of the complex data. This was compared to a tube mask pipeline (green) that used a simple tube mask that was manually defined on the echo-combined magnitude image. This mask included all the material present in the tube and the high-noise regions

\section{QSM calculation in human vessels}

Masking procedures were compared for final susceptibility map calculation through comparison of tube mask and noise mask QSM pipelines. For both pipelines, field maps were generated using nonlinear field fitting ${ }^{35,36}$ and unwrapped using Laplacian phase unwrapping. ${ }^{37}$ At this point, the pipelines diverge as background field removal and susceptibility calculation steps require masks identifying the region of interest (ROI). Local field maps were calculated with the projection-onto-dipole-fields method $^{34}$ using the unwrapped field map and a ROI mask as input, that is, tube mask for the tube mask pipeline and noise mask for the noise mask pipeline. Using the relevant local field map and mask as inputs, susceptibility maps were calculated for the tube mask and noise mask pipelines using the iterative Tikhonov approach as described for the porcine arteries. The same regularization parameter $(\alpha)$ was used for all samples and was chosen by performing L-curve optimization ${ }^{44}$ for both tube mask and noise mask pipelines in all 5 human datasets and calculating the mean of the individually optimized parameters. Similar to the porcine vessels, alternative susceptibility calculation methods (truncated k-space division, direct Tikhonov, and morphology enabled dipole inversion) were tested and found not to influence the trends and final conclusions reported for human vessels (see Supporting Information Figure S11).

\subsection{Histological analysis}

To allow histological validation, the model tissues (native, decellularized, collagen-degraded, and elastin-degraded vessel models) were fixed immediately after MR scanning for histological processing by immersing vessels in $4 \%$ formalin for 7 days at $4^{\circ} \mathrm{C}$. Stepwise dehydration was performed on the fixed tissue in ethanol to xylene, and samples were then embedded in paraffin wax and sectioned into $8 \mu \mathrm{m}$-thick slices prior to staining. 
For porcine and human vessels, Hematoxylin and Eosin (H\&E), Picrosirius red, Verhoeff's elastin, and Alcian blue staining were performed to identify the presence of smooth muscle cells, collagen, elastin, and glycosaminoglycans, respectively. ${ }^{27}$ Additionally, Alizarin Red staining was performed on human vessels to identify the presence of calcium. Histological imaging was performed using an Olympus BX41 microscope with Ocular V2.0 software (Teledyne Photometrics, Tuscon, Arizona) for the porcine models and an Aperio CS2 microscope with ImageScope software V12.3 (Leica Biosystems Imaging, Inc., Vista, California) for the human arteries. Bright-field microscopy was performed for all stains with additional polarized light microscopy on Picrosirius red to infer the orientation of collagen fibers.

For human vessels, alignment of histology to QSM was performed as follows: with anatomical features as landmarks, the first echo of the magnitude combined ME-GRE image was used to guide the identification of tissue sections in the common carotid for histological analysis. The location of the selected region on the ME-GRE image was noted and used to guide the manual alignment of the digitized whole-mount histology image to the relevant MRI slice. The final alignment of histology to MRI was refined via manual rotation and scaling of the histology image (see Figure 4 and Supporting Information Figures S2-S6)

\section{5 | Data analysis}

\subsection{1 | ROI}

For both human and porcine tissue, ROIs were manually defined for each vessel using the echo-combined MRI magnitude image. Care was taken to position ROIs within arterial tissue and avoid regions of partial volume near the edges of each vessel. This was facilitated by the high resolution of the ME-GRE data. ROIs in human vessels were limited to normal-appearing arterial tissue in the common carotid artery. This was achieved by cross-referencing the MRI defined ROI with histology and excluding regions from the final ROI that showed abnormal smooth muscle cell, collagen, elastin, GAG, or calcium content as identified by histology and defined by Stary et al. ${ }^{48}$ (see Figure 4 and Supporting Information Figures S2-S6). Using the echocombined magnitude image, ROIs were manually defined for PBS in each sample. Following ROI definition, ROI mean susceptibility values were extracted for each vessel and for PBS in each sample. To compare across samples, vessel susceptibility was referenced to $\mathrm{PBS}^{49}$ This was achieved by subtracting the mean PBS susceptibility from the mean vessel susceptibility in the same sample.

\subsection{2 | Statistical methods}

For porcine vessels, the null hypothesis of no susceptibility difference between tissue models (native, decellularized, collagen-degraded, elastin-degraded, and fixed native) was tested using a 1-way analysis of variance. If the null hypothesis was rejected, post hoc pairwise comparisons were performed using the Tukey-Kramer method. To compare the susceptibility measurements made using tube mask and noise mask pipelines in human common carotid arteries, the intraclass correlation coefficient ${ }^{50}$ was calculated. For comparison of susceptibility measurements made in human common carotid arteries with those in fixed native porcine arteries, the null hypothesis of no susceptibility difference between groups (human common tube mask $_{\text {, human common }}$ noise mask, $_{\text {, and }}$ fixed native porcine) was tested using a 1-way analysis of variance. If the null hypothesis was rejected, post hoc pairwise comparisons were performed using the Tukey-Kramer method.

\section{3 | RESULTS}

\section{1 | Histology}

\subsection{1 | Histological validation of tissue models}

Figure 3 presents histological validation of the vessel models where enzymatic digestive treatments were used to selectively remove smooth muscle cells, elastin, and collagen in selected groups of porcine carotid arteries. H\&E staining confirms the absence of smooth muscle cells in the decellularized vessels (Figure 3D); Verhoeff's elastin stain confirms the degradation of elastin in the elastindegraded vessels (Figure 3G); and Picrosirius red verifies the removal of collagen in the collagen-degraded vessels (Figure 3N,R). Furthermore, H\&E staining (Figure 3A-D) verifies that smooth muscle cell content remained intact in the non-decellularized vessels; Verhoeff's elastin staining (Figure 3E-H) verifies that elastin content was maintained in the non-elastin-degraded vessels; and Picrosirius red staining with bright field microscopy (Figure 3M-P) and polarized light microscopy (Figure 3Q-T) verifies the preservation of collagen content and orientation in the non-collagen-degraded vessels. Alcian blue staining (Figure 3I-L) showed a decrease in GAG content across all the degradation models when compared with native tissue. This may be explained by the leaching of glycosaminoglycans out of the tissue in order to maintain osmotic balance due to the presence of PBS. Although this has not been observed in arterial tissue, it has been observed in intervertebral disc and articular cartilage. ${ }^{51}$ 

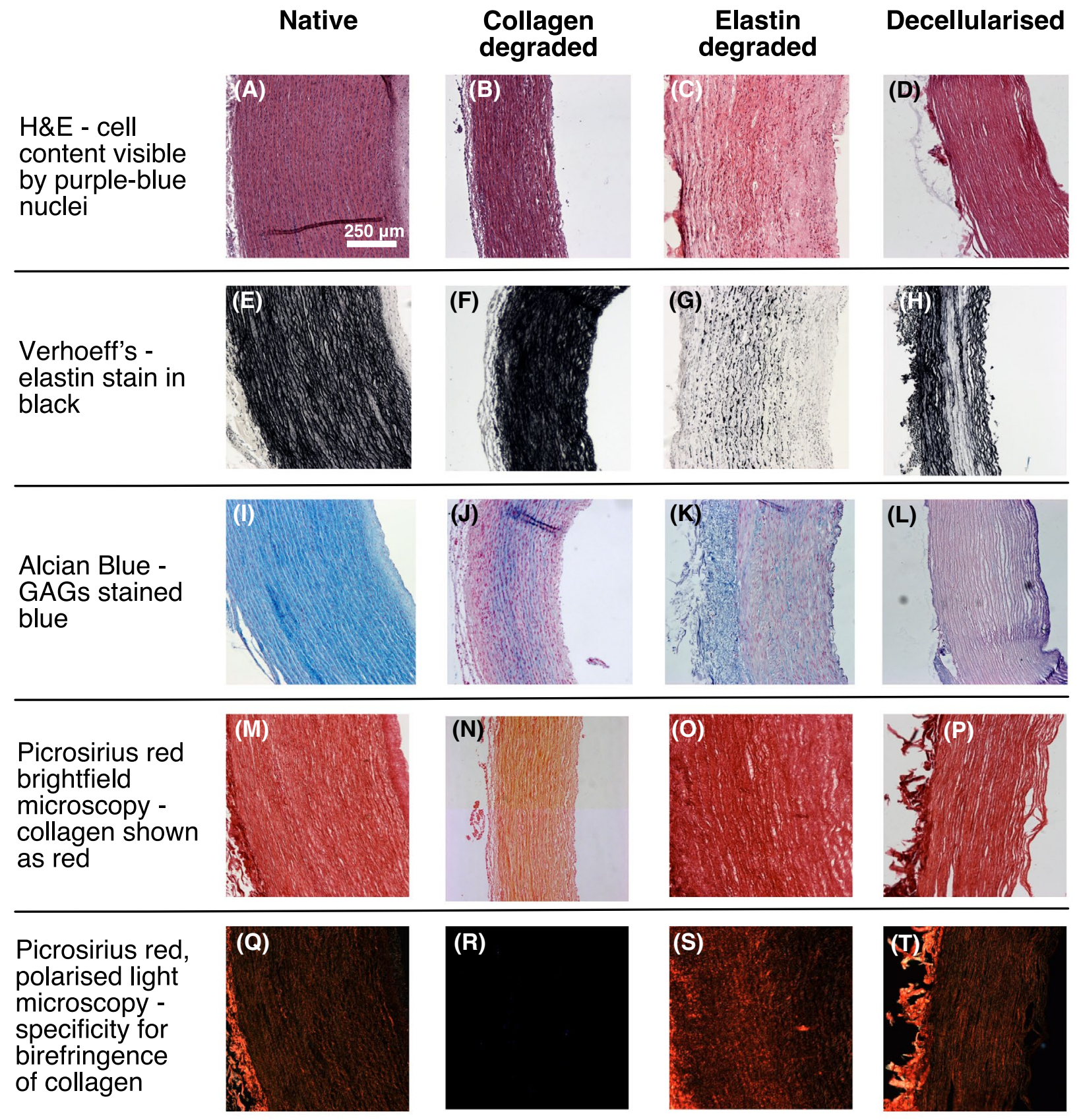

F I G U R E 3 Histological validation of porcine carotid artery tissue models. Enzymatic digestion treatments were used to selectively remove components of the arterial microstructure creating 4 different tissue models: native, collagen-degraded, elastin-degraded, and decellularized porcine carotid artery

\subsubsection{Histological validation of ROIs in cadaver vessels}

Figure 4 shows example H\&E in human common carotid artery for a representative cadaver specimen imaged in this study. Supporting Information Figures S2-S6 display histological analysis and ROI definition for each human vessel. Histology was used to guide the definition of MRI ROIs in "normal"-appearing common carotid tissue by avoiding regions of obvious abnormality or disease defined as a disruption to cell, collagen, GAG, elastin, or calcium content.

\section{2 | MRI}

\subsubsection{QSM of tissue models}

Figure 5 presents susceptibility and $\mathrm{R}_{2}^{*}$ relaxometry maps for each of the vessel models. Susceptibility maps produced 
(A) Magnitude Image (ME-GRE TE ) $_{1}$

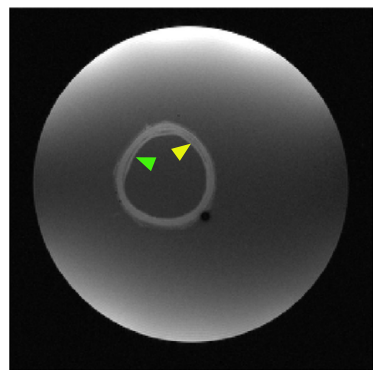

(B) Region of Interest

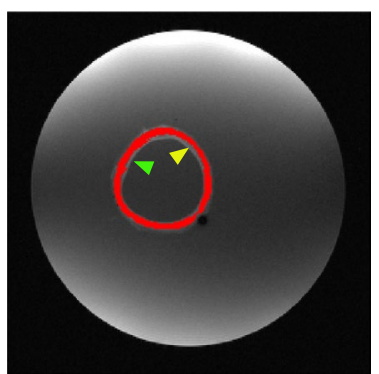

(C) H\&E Histology

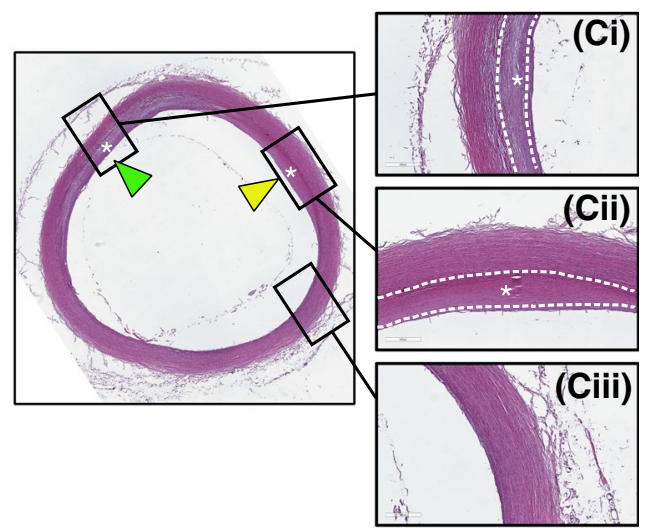

F I G U RE 4 Example of MRI ROI definition in normal-appearing human common carotid. ME-GRE images (A) with ROI overlay (B) are displayed alongside H\&E histology (c). H\&E histology identifies regions of intimal thickening (Ci) and (Cii) and healthy-appearing vessel microstructure (Ciii). Abnormal regions are excluded from ROI definition on the magnitude images (green and yellow arrows (B)). Detailed comparison of MRI ROIs and histological analysis for each vessel are shown in Supporting Information Figures S2-S6. GRE, gradient echo; H\&E, Hematoxylin and Eosin; ROI, region of interest

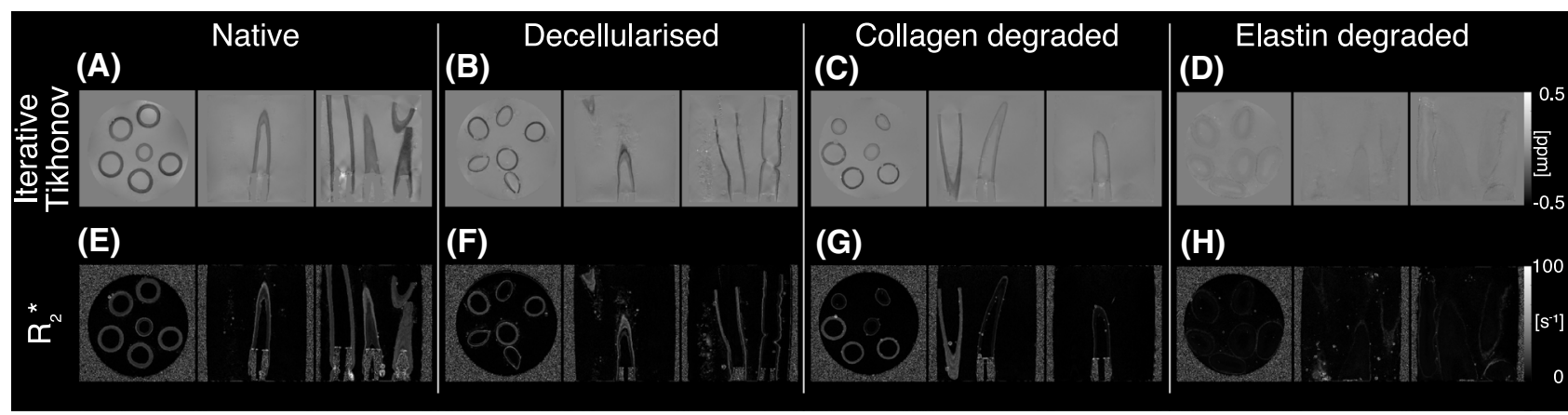

F I G URE 5 Susceptibility maps produced for the different porcine carotid tissue models (native, decellularized, collagen-degraded, and elastin-degraded) using the iterative Tikhonov approach (A-D) alongside maps of $\mathrm{R}_{2}^{*}(\mathrm{E}-\mathrm{H})$. Equivalent susceptibility maps calculated with alternative susceptibility calculation methods (truncated k-space division, direct Tikhonov, and morphology enabled dipole inversion) are compared in Supporting Information Figure S8

using the iterative Tikhonov method are presented in 3 different viewing planes (axial, coronal, and sagittal in the context of the tube, which was placed with its long axis parallel to the orientation of $\mathrm{B}_{0}$ ) for qualitative comparison of image quality. The regularization parameter used for susceptibility map calculation in porcine vessels was $\alpha=0.02$, as determined by taking the mean of the L-curve optimization ${ }^{44}$ across the 5 samples (Supporting Information Figure S7).

Qualitative differences are apparent when comparing the susceptibility maps of different vessel models. The susceptibility of native (Figure 5A) vessels appear the most diamagnetic, with the susceptibility of decellularized (Figure 5B) and collagen-degraded (Figure 5C) vessels appearing elevated in comparison while maintaining contrast with the background fluid. The susceptibility of the elastin-degraded vessels (Figure 5D) appear the most elevated, with almost no susceptibility difference with the surrounding fluid. Qualitatively, $\mathrm{R}_{2}^{*}$ relaxometry maps demonstrated similar but inverse trends to the susceptibility maps, with $\mathrm{R}_{2}^{*}$ of native vessels (Figure 5E) appearing highest and $\mathrm{R}_{2}^{*}$ of elastin-degraded vessels (Figure 5H) appearing lowest and similar to that of surrounding background fluid.

As a quantitative comparison, Figure 6 shows box plots of the susceptibility and $\mathrm{R}_{2}^{*}$ values measured in each vessel ( $\mathrm{n}=6$ per model) and grouped by tissue model. The mean ( \pm standard deviation [SD]) susceptibility of PBS measured across the 5 samples was $0.0219 \pm 0.0121 \mathrm{ppm}$. Susceptibility and $\mathrm{R}_{2}^{*}$ measurements in fixed native porcine carotid artery $(n=6)$ are presented for later comparison with human vessels. The baseline magnetic susceptibility of native porcine arteries was found to be diamagnetic with a mean value of $-0.1820 \mathrm{ppm}$, and vessel susceptibilities ranged from $-0.2346 \mathrm{ppm}$ to $-0.0092 \mathrm{ppm}$ across all tissue models. The mean susceptibility of the tissue models $\left(\chi_{\text {elastin-degraded }}=\right.$ $-0.0163 \mathrm{ppm} ; \chi_{\text {collagen-degraded }}=-0.1158 \mathrm{ppm} ; \chi_{\text {decellularized }}=$ $\left.-0.1379 \mathrm{ppm} ; \chi_{\text {fixed native }}=-0.2199 \mathrm{ppm}\right)$ exhibited the 

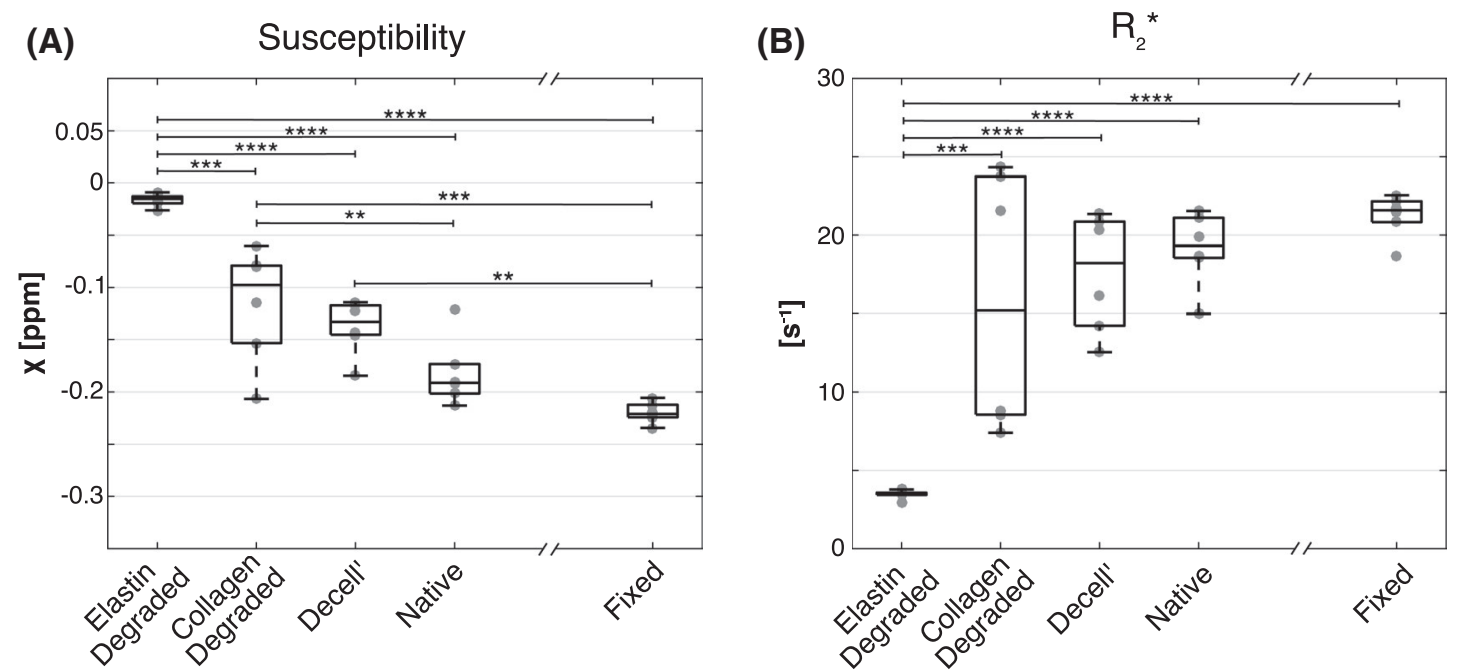

F I G URE 6 Box plots comparing measurements extracted from vessels $(n=6)$ of each tissue model for QSM (A) and R ${ }_{2}^{*}$ maps $(B)$. Gray data points represent susceptibility measurements in individual vessels. Measurements compared using ANOVA and post hoc test $(* P<.05$, $* * P<$ $.01, * * * P<.001, * * * * P<.0001)$. Equivalent measurements made using alternative susceptibility calculation methods are presented in Supporting Information Figure S10. ANOVA, analysis of variance

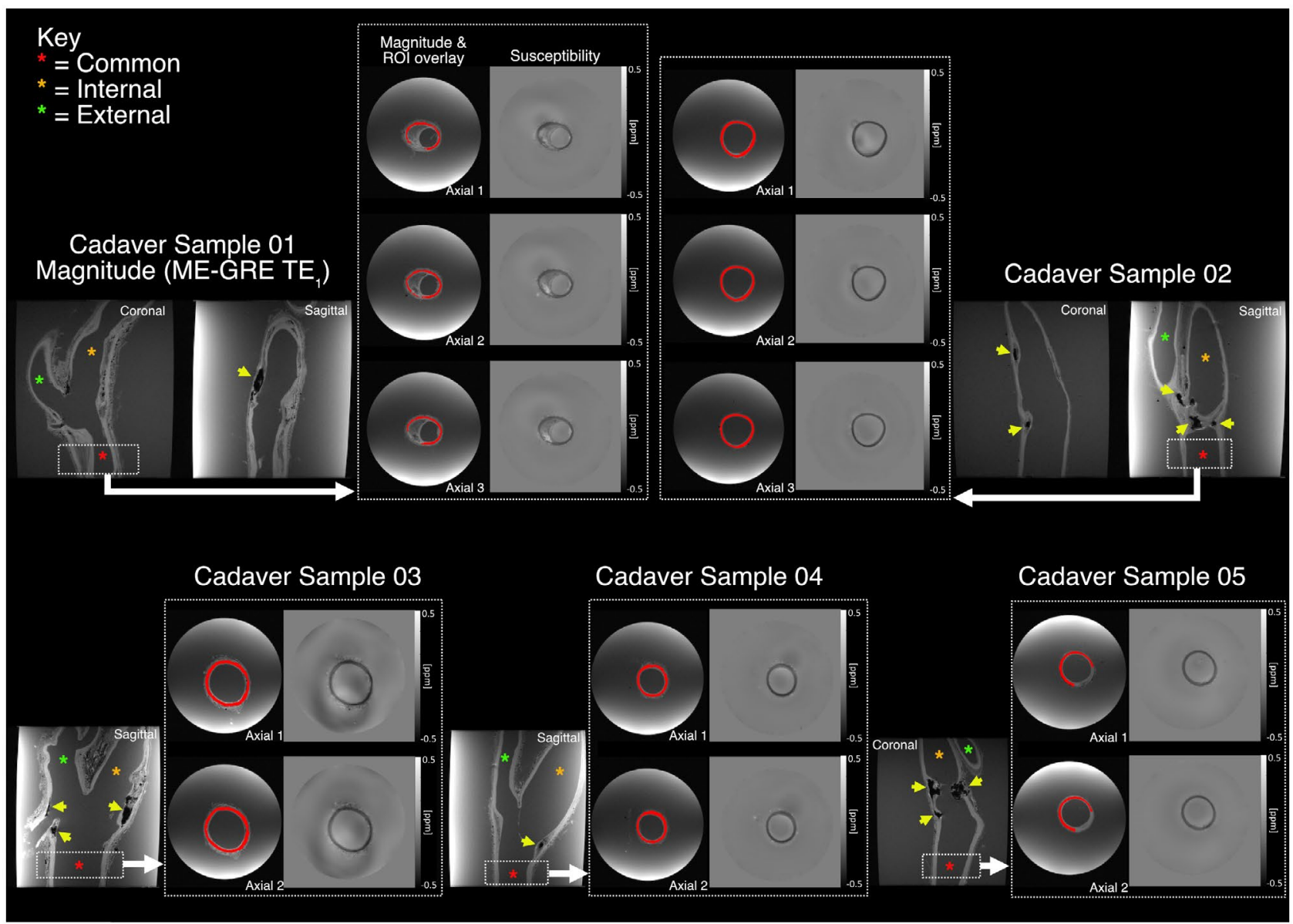

F I G U RE 7 Measurements in human cadaver samples were confined to "normal" common carotid artery tissue with no areas of atherosclerotic disease. The location of the common carotid ROI is displayed on first-echo magnitude images from the ME-GRE acquisition. Common (red asterisk), internal (yellow asterisk), and external (green asterisk) carotid arteries can be seen in the sagittal/coronal magnitude images. Representative QSM axial images in the common carotid ROI are presented alongside first-echo magnitude axial images with red ROI overlaid. ME, multi-echo 
following trend: $\chi_{\text {elastin-degraded }}>\chi_{\text {collagen-degraded }}>\chi_{\text {decellularized }}$ $>\chi_{\text {native }}>\chi_{\text {fixed native. }}$ Significant differences were detected between the susceptibility of the vessel models (analysis of variance, $P<.001$ ). Post hoc pairwise comparisons revealed the susceptibility of collagen-degraded vessels to be significantly higher than native vessels $(P<.01)$, the susceptibility of elastin-degraded vessels to be significantly higher than all other vessel groups $(P<.001)$, and the susceptibility of fixed native vessels to be significantly lower than both decellularized $(P<.01)$ and collagen-degraded vessels $(P<$ .001). Comparing vessel-wise measurements of $\mathrm{R}_{2}^{*}$, the null hypothesis was rejected $(P<.001)$, indicating differences between groups. Post hoc testing revealed significantly lower $\mathrm{R}_{2}^{*}$ in elastin-degraded vessels compared to all other vessel models $(P<.001)$.

\subsubsection{QSM of human common carotids}

In the human carotid arteries, regions of advanced disease were seen close to the bifurcation in all 5 vessels scanned in this study (Figure 7, yellow arrows). These heavily diseased regions contained structures with little or no signal-likely attributable to the presence of calcification or hemorrhage. For a representative vessel, susceptibility maps are presented for tube mask and noise mask pipelines, with the region where the ROI was defined indicated in red (Supporting Information Figure S9). It can be seen that human carotid vessels exhibited pronounced streaking artefacts attributed to the inclusion of low SNR regions in the QSM calculation (tube mask). Streaking was visibly reduced in the susceptibility maps produced using the noise mask pipeline (Supporting Information Figure S9). The optimal regularization parameter was determined as $\alpha=0.02$ using L-curve optimization, remaining the same as that used for the porcine vessels (Supporting Information Figure S7).

Figure 8 shows susceptibility measurements in human common carotid arteries. The mean susceptibility of human common carotid was $-0.1898 \pm 0.0253 \mathrm{ppm}$ using the noise mask pipeline and $-0.2007 \pm 0.0183$ ppm using the tube mask pipeline. This is in comparison to the fixed native porcine carotid arteries, which had a mean susceptibility of $-0.2199 \pm$ $0.0100 \mathrm{ppm}$. Figure 8A directly compares common carotid susceptibility measurements between the noise mask and tube mask pipelines, with an intraclass correlation coefficient value of $0.88(P<.05)$ being obtained between the pipelines. Figure $8 \mathrm{~B}$ displays box plots of the mean susceptibility in each vessel using the noise mask and tube mask pipelines compared with those in fixed native porcine carotid arteries. The null hypothesis was not rejected $(P>.05)$; however, the $P$ value of 0.050 was close to the significance level of 0.05 . No significant difference was seen in the PBS susceptibility
(A)

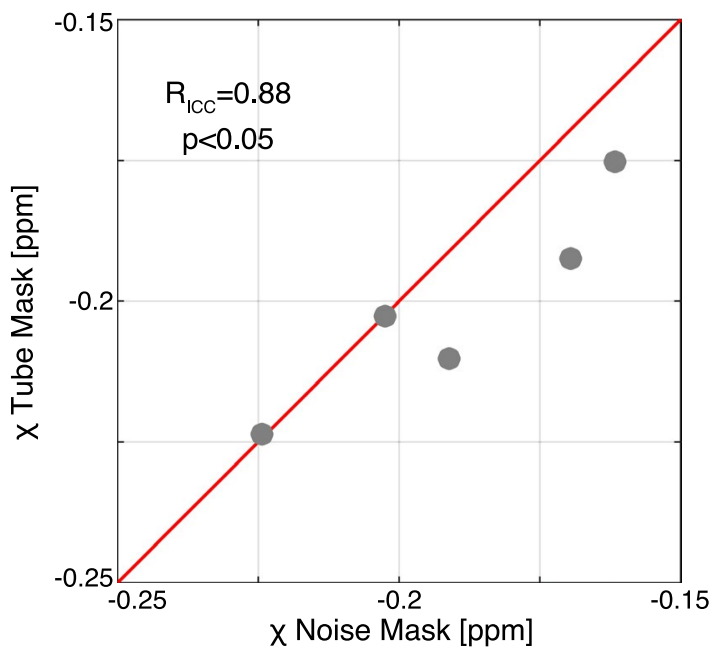

(B)

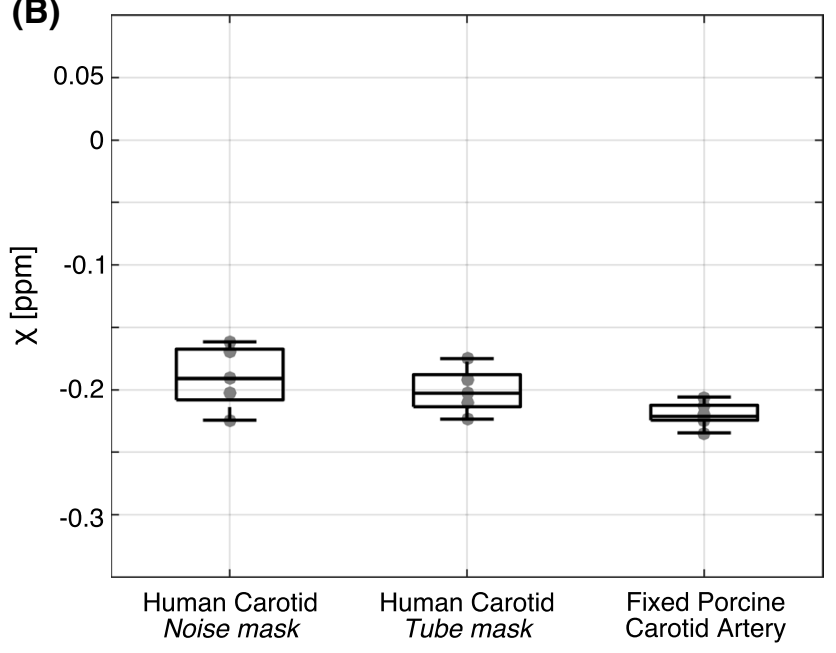

F I G URE 8 Magnetic susceptibility of human common carotid. The effect of masking out noisy regions on mean susceptibility values in the cadaver common carotid ROI are shown in (A). Agreement between different masking pipelines was excellent using the following criteria: poor, < 0.4; fair, 0.41-0.59; good, 0.60-0.74; and excellent, $>0.75$. $^{52}$ Box plots comparing susceptibility measurements in cadaver common carotid arteries with those in fixed porcine carotid arteries is shown in (B). Susceptibility values were compared using ANOVA, and no significant difference was seen between groups $(P=.0501)$. Equivalent measurements made using alternative susceptibility calculation methods are presented in Supporting Information Figure S11

reference between the human and porcine vessels (analysis of variance, $P>.05$ ).

\section{4 | DISCUSSION}

\section{1 | Main findings}

In this study, we demonstrate for the first time the sensitivity of magnetic susceptibility, measured using QSM, to the 
microstructural composition of arterial tissue. Vessels with different microstructural compositions were generated by applying different enzymatic digestion treatments to ex vivo porcine carotid arteries. For each model (decellularized, collagendegraded, and elastin-degraded), 6 vessels were imaged using a high-resolution QSM protocol and compared to untreated (native) and fixed native porcine carotid arteries (Figure 5). The microstructural composition of each vessel model was validated using histology (Figure 3), and statistically significant differences were found between the group mean susceptibility of porcine vessels with different microstructural compositions (Figure 6). Post hoc statistical testing revealed significantly higher susceptibility in collagen-degraded vessels compared to native vessels, whereas no such difference was present in equivalent $\mathrm{R}_{2}^{*}$ relaxometry measurements. Significantly lower susceptibility was measured in fixed native vessels compared to both decellularized and collagen-degraded vessels, with no such statistical differences existing in the equivalent $\mathrm{R}_{2}^{*}$ measurements. Significantly higher susceptibility and $\mathrm{R}_{2}^{*}$ were measured in elastin-degraded vessels compared to all other vessel models. However, the measurements made in the elastin-degraded vessels were confounded by partial volume effects with PBS due to a large increase in extracellular space caused by elastin removal (further discussion in Section 4.2.2). This suggests that QSM offers improved sensitivity to the microstructural composition of arterial vessels, in particular collagen, when compared to equivalent $\mathrm{R}_{2}^{*}$ measurements.

To provide a comparison with human tissue, susceptibility was measured in ex vivo human common carotid arteries using the same high-resolution QSM pipeline (Figure 7). Regions of advanced disease were present in these vessels, leading to streaking artefacts in the susceptibility maps. Exclusion of these low SNR regions resulted in a qualitative improvement in the susceptibility maps (Figure S9). However, using intraclass correlation coefficient, "excellent" agreement ${ }^{52}$ was found between human common carotid measurements extracted from susceptibility maps that were calculated using pipelines, which included and excluded the low SNR diseased regions (Figure 8A). One explanation for this agreement is that the common carotid is positioned far enough away from the streaks (originating in the bifurcation) that they have very little effect on the mean susceptibility measured in that region. An example of this can be seen in Figure S9. Statistical testing revealed no significant differences between magnetic susceptibilities measured in fixed human common carotid arteries and fixed porcine vessels (Figure 8B).

\section{2 | Susceptibility and arterial microstructure}

Experimental results from porcine vessel models (Figure 6) suggest tissue susceptibility, measured using QSM, is sensitive to the microstructural composition of arterial vessels. The baseline magnetic susceptibility of native porcine arteries was found to be diamagnetic with a mean value of $-0.1820 \mathrm{ppm}$ and agrees well with the value of $-0.25 \pm 0.14 \mathrm{ppm}$ reported for popliteal artery wall in vivo. ${ }^{24}$

\subsection{1 | Collagen}

Collagen present in arterial tissue is primarily of type I and type III. ${ }^{53}$ At a molecular level, these collagen types exhibit the characteristic triple helical structure, leading to a diamagnetic susceptibility as described by Wei et al. ${ }^{24}$ Compared to native vessels, a significantly higher susceptibility was measured in collagen-degraded vessels $(\Delta \chi=$ $0.0662 \mathrm{ppm}$ ), whereas no such significant difference was apparent in the equivalent $\mathrm{R}_{2}^{*}$ relaxometry measurements. This agrees well with studies of collagen susceptibility in articular cartilage ${ }^{24,54}$ and liver fibrosis, ${ }^{21}$ which observed collagen as strongly diamagnetic. Although articular cartilage degradation associated with collagen loss has been shown to cause changes in measured susceptibility in vivo, ${ }^{55}$ ex vivo studies of articular cartilage failed to detect differences in the susceptibility of collagen-degraded samples. ${ }^{22}$ The differences in the results found in ex vivo collagen-degraded articular cartilage and arterial vessels may well be explained by differences in experimental setup, susceptibility reference material, and enzymatic digestion treatments. It is important to note that collagen was completely removed from the samples imaged in this study (Figure 3N,R), and PBS was used as a consistent susceptibility reference between samples.

\subsection{2 | Elastin}

At a molecular level, elastin is highly cross-linked, with these cross-links existing in a $\alpha$-helical conformation. ${ }^{56}$ Results presented in this study suggest elastin to have a diamagnetic susceptibility. However, no specific measurements of elastin magnetic susceptibility have been reported prior to this study. Significant differences were seen between the measured susceptibility of elastin-degraded vessels and all other vessel models, including native and fixed native vessels. The elastin-degraded group also demonstrated the largest deviation in measured susceptibility from native vessels. However, as evidenced histologically by H\&E staining (Figure 3A,C), the removal of elastin results in a less compact microstructural arrangement of tissue when compared to native vessel histology. This suggests that the removal of elastin results in an increased extracellular space, allowing the penetration of surrounding PBS into the tissue microstructure. This is supported by the visible increase in size 
of the vessels and the distinct lack of contrast seen between elastin-degraded vessels and the surrounding PBS in QSM (Figure 5D). Therefore, it is difficult to conclusively assess the contribution of elastin to vessel susceptibility from the tissue model presented here.

\subsection{3 | Smooth muscle cells}

Considerable overlap is seen between the measured susceptibilities of decellularized and native vessels. The slightly higher group mean susceptibility of decellularized vessels was not significantly different from that of native vessels (Figure 6).

The results from all these porcine artery models suggest that QSM is most sensitive to detecting changes in arterial collagen. Further work is required to investigate the integrity of the elastin model and the resulting abolition, following elastin degradation, of the diamagnetic susceptibility found in native porcine arteries. An alternative approach to characterizing the magnetic susceptibility of elastin is to use $3 \mathrm{D}$ printed tissue scaffolds where the density of elastin can be tightly controlled. ${ }^{57}$

Although all the enzymatic digestion treatments in this study were performed on porcine carotids, the study did not control for the differences between proximal or distal sections of the porcine vessels. A gradual change in microstructure exists across the length of the carotid vessel, with more proximal regions being more elastic and distal regions being more muscular. ${ }^{15}$ This variation likely explains the susceptibility and $\mathrm{R}_{2}^{*}$ contrast differences between arteries in Figure 5 (i.e., top 3 vessels in the collagen model and variation between decellularized vessels); however. the digestive treatments performed were designed and confirmed to selectively remove all of a specific microstructural component from a given vessel.

\subsection{4 | Susceptibility anisotropy}

Although this study has demonstrated the sensitivity of QSM to arterial microstructure, specific components such as collagen are known to possess $\mathrm{B}_{0}$ orientation-dependent magnetic susceptibility. ${ }^{21-23}$ In this study, all vessels were imaged at the same orientation to the main magnetic field (long axis of the vessel parallel to $\mathrm{B}_{0}$ ). Because arterial collagen and elastin fibers are circumferentially arranged, these fibers will be oriented at $90^{\circ}$ to $\mathrm{B}_{0}$, facilitating consistent comparison of measured susceptibility within and between vessels. Because QSM assumes isotropic susceptibility, further work is required to assess the susceptibility anisotropy of arterial tissue and the anisotropic contributions of its microstructural components, collagen in particular. ${ }^{54}$

\section{3 | Human artery}

\subsubsection{Comparison of human and porcine vessels}

From Figure 8B, variability of vessel susceptibility across the group is visibly lower for porcine vessels compared to the human samples. This may be partially attributed to the low variability in the age of the pigs compared to the human subjects but also to differences between fixation and embalming, respectively. Porcine vessels were imaged directly after fixation, whereas time from embalming was not controlled for in human vessels. Evia et al. reported no systematic change in magnetic susceptibility of fixed post-mortem brain over a period of 6 weeks, ${ }^{58}$ but changes in susceptibility outside this timeframe may be possible. Although specific information regarding time from embalming to scanning is not available for the human vessels, imaging of all vessels included in this study occurred after this 6-week time frame. The variability in susceptibility seen between human vessels could also be due to biological variability (e.g., age, sex, body size, and disease status), as well as its effect on the embalming protocol.

Despite this variability, there was no statistically significant difference measured between the magnetic susceptibility of human and porcine arterial tissue. This suggests that the sensitivity of magnetic susceptibility to microstructural composition, demonstrated in porcine arterial tissue, is also likely to be found in human arterial tissue. This provides a promising springboard for studies seeking to translate QSM for use in characterizing human carotid arteries and disease.

Significant differences between vessel mean susceptibility values calculated using different techniques were seen in this study (Figure S11). However, the trend of degradation-dependent susceptibility differences was identical in use of all susceptibility calculation methods (Figure S10).

\subsection{2 | Fixed porcine tissue}

The group mean susceptibility was slightly lower in fixed tissue $(\chi=-0.2199 \mathrm{ppm})$ compared to native vessels $(\chi=$ $-0.1820 \mathrm{ppm})$, although this difference was not statistically significant (Figure 6). As noted by Wei et al, ${ }^{21}$ fixation alters tissue microstructure through crosslinking of proteins, and differences in measured susceptibility are not surprising. Differences in susceptibility between in vivo and fixed ex vivo mouse brain have been reported, ${ }^{59}$ and it has been noted that changes in tissue relaxation times that accompany fixation could impact QSM measurements of susceptibility. ${ }^{26}$ 


\subsection{Conclusion, impact, and future work}

In diseased arterial tissue, key components of the tissue microstructure, such as collagen fibers, smooth muscle cells, and elastic lamina, become disrupted, impairing vessel function. ${ }^{60} \mathrm{~A}$ number of recent studies have demonstrated the application of QSM for imaging carotid plaque in vivo, highlighting that many technical challenges associated with imaging carotid arteries using QSM in a clinical setting can be overcome. ${ }^{9-13}$ These studies have demonstrated marked improvement in the depiction of intraplaque hemorrhage and calcification in vivo but have largely ignored differences in regional plaque susceptibility that may be driven by compositional variations in the microstructure of fibrotic plaque tissue. Results from this study highlight that QSM is sensitive to the microstructural composition of arterial tissue, and with further development it has the potential to offer unique insight into the onset and progression of carotid atherosclerosis. Such characterization of carotid plaques has the potential to improve the assessment of stroke risk using MRI $^{61}$ and could complement existing MRI methods capable of detecting downstream hemodynamic alterations. ${ }^{62,63}$ Future work will focus on QSM of diseased arterial tissue ex vivo, using the insights from this study as a basis to fully characterize the susceptibility contributions from intraplaque hemorrhage, calcifications, lipid, and tissue microstructure in heterogenous atherosclerotic plaques.

\section{ACKNOWLEDGMENT}

This research was funded by the European Research Council (ERC) under the European Union's Horizon 2020 research innovation programme (grant 637674). The authors thank and acknowledge the Department of Anatomy, Royal College of Surgeons in Ireland (Professor Clive Lee and Bob Dalchan), for supporting this work. Human tissue was used with approval from the Department of Anatomy, Royal College of Surgeons in Ireland institutional review board. Open access funding provided by IReL. KS acknowledges funding from ERC Consolidator Grant DiSCo MRI SFN 770939.

\section{DATA AVAILABILITY STATEMENT}

The imaging data for this study are available from the corresponding author upon reasonable request. Tools used for QSM calculation are openly available through the MEDI toolbox (http://weill.cornell.edu/mri/pages/qsm.html) and UCL's XIP repository (https://xip.uclb.com/i/software/mri qsm_tkd.html).

\section{ORCID}

Brooke Tornifoglio (D) https://orcid.

org/0000-0002-4359-9012

Karin Shmueli (D) https://orcid.org/0000-0001-7520-2975

Caitríona Lally (iD https://orcid.org/0000-0003-4141-1685

\section{TWITTER}

Caitríona Lally @ @trionalally

\section{REFERENCES}

1. Gupta A, Gialdini G, Giambrone AE, et al. Association between nonstenosing carotid artery plaque on MR angiography and acute ischemic stroke. JACC Cardiovasc Imaging. 2016;9:1228-1229.

2. Zhao X, Hippe DS, Li R, et al. Prevalence and characteristics of carotid artery high-risk atherosclerotic plaques in Chinese patients with cerebrovascular symptoms: a Chinese atherosclerosis risk evaluation II study. J Am Heart Assoc. 2017;6:1-11.

3. Alpert JN. Extracranial carotid artery: current concepts of diagnosis and management. Tex Heart Inst J. 1991;18:93-97.

4. Falk E. Why do plaques rupture? Circulation. 1992;86:III30-III42.

5. Brinjikji W, Huston J, Rabinstein AA, Kim GM, Lerman A, Lanzino G. Contemporary carotid imaging: from degree of stenosis to plaque vulnerability. J Neurosurg. 2016;124:27-42.

6. Deistung A, Schweser F, Reichenbach JR. Overview of quantitative susceptibility mapping. NMR Biomed. 2017;30. https://doi. org/10.1002/nbm.3569

7. Wang Y, Liu T. Quantitative susceptibility mapping (QSM): decoding MRI data for a tissue magnetic biomarker. Magn Reson Med. 2015;73:82-101.

8. Haacke EM, Liu S, Buch S, Zheng W, Wu D, Ye Y. Quantitative susceptibility mapping: current status and future directions. Magn Reson Imaging. 2015;33:1-25.

9. Ikebe Y, Ishimaru H, Imai H, et al. Quantitative susceptibility mapping for carotid atherosclerotic plaques: a pilot study. Magn Reson Med Sci. 2020;19:135-140.

10. Azuma M, Maekawa K, Yamashita A, et al. Characterization of carotid plaque components by quantitative susceptibility mapping. Am J Neuroradiol. 2020;41:310-317.

11. Ruetten PPR, Cluroe AD, Usman A, Priest AN, Gillard JH, Graves MJ. Simultaneous MRI water-fat separation and quantitative susceptibility mapping of carotid artery plaque pre- and post-ultrasmall superparamagnetic iron oxide-uptake. Magn Reson Med. 2020;84:686-697.

12. Wang C, Zhang Y, Du J, et al. Quantitative susceptibility mapping for characterization of intraplaque hemorrhage and calcification in carotid atherosclerotic disease. J Magn Reson Imaging. 2020;52:534-541.

13. Nguyen TD, Wen Y, Du J, et al. Quantitative susceptibility mapping of carotid plaques using nonlinear total field inversion: initial experience in patients with significant carotid stenosis. Magn Reson Med. 2020;84:1501-1509.

14. Chen W, Zhu W, Kovanlikaya IIhami, et al. Intracranial calcifications and hemorrhages: characterization with quantitative susceptibility mapping. Radiology. 2014;270:496-505.

15. Rhodin JAG. Architecture of the vessel wall. In: Comprehensive Physiology. Terjung R, ed. Supplement 7. Handbook of Physiology, The Cardiovascular System, Vascular Smooth Muscle. Hoboken, NJ: John Wiley \& Sons; 1980:1-31.

16. Holzapfel GA, Gasser TC, Ogden RW. A new constitutive framework for arterial wall mechanics and a comparative study of material models. J Elast. 2000;61:1-48.

17. Hansson GK, Libby P, Tabas I. Inflammation and plaque vulnerability. J Intern Med. 2015;278:483-493.

18. Liu C. Susceptibility tensor imaging. Magn Reson Med. 2010;63:1471-1477. 
19. Lee J, Shmueli K, Fukunaga M, et al. Sensitivity of MRI resonance frequency to the orientation of brain tissue microstructure. Proc Natl Acad Sci U S A. 2010;107:5130-5135.

20. Li J, Lin H, Liu T, et al. Quantitative susceptibility mapping (QSM) minimizes interference from cellular pathology in R2* estimation of liver iron concentration. J Magn Reson Imaging. 2018;48:1069-1079.

21. Wei H, Decker K, Nguyen H, et al. Imaging diamagnetic susceptibility of collagen in hepatic fibrosis using susceptibility tensor imaging. Magn Reson Med. 2020;83:1322-1330.

22. Nykänen O, Rieppo L, Töyräs J, et al. Quantitative susceptibility mapping of articular cartilage: ex vivo findings at multiple orientations and following different degradation treatments. Magn Reson Med. 2018;80:2702-2716.

23. Wei H, Gibbs E, Zhao P, et al. Susceptibility tensor imaging and tractography of collagen fibrils in the articular cartilage. Magn Reson Med. 2017;78:1683-1690.

24. Wei H, Dibb R, Decker K, et al. Investigating magnetic susceptibility of human knee joint at 7 Tesla. Magn Reson Med. 2017;78:1933-1943.

25. Xie L, Dibb R, Cofer GP, et al. Susceptibility tensor imaging of the kidney and its microstructural underpinnings. Magn Reson Med. 2015;73:1270-1281.

26. Dibb R, Qi Y, Liu C. Magnetic susceptibility anisotropy of myocardium imaged by cardiovascular magnetic resonance reflects the anisotropy of myocardial filament $\alpha$-helix polypeptide bonds. $J$ Cardiovasc Magn Reson. 2015;17:1-14.

27. Tornifoglio B, Stone AJ, Johnston RD, Shahid SS, Kerskens C, Lally C. Diffusion tensor imaging and arterial tissue: establishing the influence of arterial tissue microstructure on fractional anisotropy, mean diffusivity and tractography. Sci Rep. 2020;10:1-12.

28. Müller-Schweinitzer E. Cryopreservation of vascular tissues. Organogenesis. 2009;5:97-104.

29. Pegg DE, Wusteman MC, Boylan S. Fractures in cryopreserved elastic arteries. Cryobiology. 1997;34:183-192.

30. Campbell EM, Cahill PA, Lally C. Investigation of a smalldiameter decellularised artery as a potential scaffold for vascular tissue engineering; biomechanical evaluation and preliminary cell seeding. J Mech Behav Biomed Mater. 2012;14:130-142.

31. Dusek P, Madai VI, Huelnhagen T, et al. The choice of embedding media affects image quality, tissue R2*, and susceptibility behaviors in post-mortem brain MR microscopy at 7.0T. Magn Reson Med. 2019;81:2688-2701.

32. Larsson EG, Erdogmus D, Yan R, Principe JC, Fitzsimmons JR SNR-optimality of sum-of-squares reconstruction for phased-array magnetic resonance imaging. J Magn Reson. 2003;163:121-123.

33. Bernstein MA, Grgic M, Brosnan TJ, Pelc NJ. Reconstructions of phase contrast, phased array multicoil data. Magn Reson Med. 1994;32:330-334.

34. Pei M, Nguyen TD, Thimmappa ND, et al. Algorithm for fast monoexponential fitting based on auto-regression on linear operations (ARLO) of data. Magn Reson Med. 2015;73:843-850.

35. Liu T, Wisnieff C, Lou M, Chen W, Spincemaille P, Wang Y. Nonlinear formulation of the magnetic field to source relationship for robust quantitative susceptibility mapping. Magn Reson Med. 2013;69:467-476.

36. Cornell MRI Research Lab. Quantitative Susceptibility Mapping (QSM) Toolbox software package. Available at: http://weill.corne 1l.edu/mri/pages/qsm.html. Accessed August 2019.
37. Schofield MA, Zhu Y. Fast phase unwrapping algorithm for interferometric applications. Opt Lett. 2003;28:1194-1196.

38. de Rochefort L, Liu T, Kressler B, et al. Quantitative susceptibility map reconstruction from MR phase data using Bayesian regularization: validation and application to brain imaging. Magn Reson Med. 2010;63:194-206.

39. Liu T, Khalidov I, de Rochefort L, et al. A novel background field removal method for MRI using projection onto dipole fields (PDF). NMR Biomed. 2011;24:1129-1136.

40. Schweser F, Robinson SD, de Rochefort L, Li W, Bredies K. An illustrated comparison of processing methods for phase MRI and QSM: removal of background field contributions from sources outside the region of interest. NMR Biomed. 2017;30. https://doi. org/10.1002/nbm.3604

41. Langkammer C, Schweser F, Shmueli K, et al. Quantitative susceptibility mapping: report from the 2016 reconstruction challenge. Magn Reson Med. 2018;79:1661-1673.

42. Karsa A, Punwani S, Shmueli K. An optimized and highly repeatable MRI acquisition and processing pipeline for quantitative susceptibility mapping in the head-and-neck region. Magn Reson Med. 2020;84:3206-3222.

43. Schweser F, Deistung A, Sommer K, Reichenbach JR. Toward online reconstruction of quantitative susceptibility maps: superfast dipole inversion. Magn Reson Med. 2013;69:1581-1593.

44. Hansen PC, O'Leary DP. The use of the L-curve in the regularization of discrete Ill-posed problems. SIAM J Sci Comput. 1993;14:1487-1503.

45. Shmueli K, de Zwart JA, van Gelderen P, Li TQ, Dodd SJ, Duyn JH. Magnetic susceptibility mapping of brain tissue in vivo using MRI phase data. Magn Reson Med. 2009;62:1510-1522.

46. Kressler B, de Rochefort L, Liu T, Spincemaille P, Jiang Q, Wang Y. Nonlinear regularization for per voxel estimation of magnetic susceptibility distributions from MRI field maps. IEEE Trans Med Imaging. 2010;29:273-281.

47. Liu J, Liu T, de Rochefort L, et al. Morphology enabled dipole inversion for quantitative susceptibility mapping using structural consistency between the magnitude image and the susceptibility map. Neuroimage. 2012;59:2560-2568.

48. Stary HC, Chandler AB, Dinsmore RE, et al. A definition of advanced types of atherosclerotic lesions and a histological classification of atherosclerosis: a report from the Committee on Vascular Lesions of the Council on Arteriosclerosis, American Heart Association. Circulation. 1995;92:1355-1374.

49. Straub S, Schneider TM, Emmerich J, et al. Suitable reference tissues for quantitative susceptibility mapping of the brain. Magn Reson Med. 2017;78:204-214.

50. Shrout PE, Fleiss JL. Intraclass correlations: uses in assessing rater reliability. Psychol Bull. 1979;86:420-428.

51. Johnson ZI, Shapiro IM, Risbud MV. Extracellular osmolarity regulates matrix homeostasis in the intervertebral disc and articular cartilage: evolving role of TonEBP. Matrix Biol. 2014;40: $10-16$.

52. Cicchetti DV. The precision of reliability and validity estimates re-visited: distinguishing between clinical and statistical significance of sample size requirements. J Clin Exp Neuropsychol. 2001;23:695-700

53. Chow MJ, Turcotte R, Lin CP, Zhang Y. Arterial extracellular matrix: a mechanobiological study of the contributions and interactions of elastin and collagen. Biophys J. 2014;106:2684-2692. 
54. Dibb R, Xie L, Wei H, Liu C. Magnetic susceptibility anisotropy outside the central nervous system. NMR Biomed. 2017;30. https:// doi.org/10.1002/nbm.3544

55. Wei H, Lin H, Qin LE, et al. Quantitative susceptibility mapping of articular cartilage in patients with osteoarthritis at 3T. J Magn Reson Imaging. 2019;49:1665-1675.

56. Anwar RA. Elastin: a brief review. Biochem Educ. 1990;18:162-166.

57. Berglund JD, Nerem RM, Sambanis A. Incorporation of intact elastin scaffolds in tissue-engineered collagen-based vascular grafts. Tissue Eng. 2004;10:1526-1535.

58. Evia AM, Kotrotsou A, Tamhane AA, et al. Ex-vivo quantitative susceptibility mapping of human brain hemispheres. PLoS One. 2017;12:e0188395.(12):1-16.

59. Wang N, Zhuang J, Wei H, Dibb R, Qi Y, Liu C. Probing demyelination and remyelination of the cuprizone mouse model using multimodality MRI. J Magn Reson Imaging. 2019;50:1852-1865.

60. Humphrey JD, Eberth JF, Dye WW, Gleason RL. Fundamental role of axial stress in compensatory adaptations by arteries. J Biomech. 2009;42:1-8.

61. Baradaran H, Gupta A. Brain imaging biomarkers of carotid artery disease. Ann Transl Med. 2020;8:1277.

62. Cho J, Zhang S, Kee Y, et al. Cluster analysis of time evolution (CAT) for quantitative susceptibility mapping (QSM) and quantitative blood oxygen level-dependent magnitude (qBOLD)-based oxygen extraction fraction (OEF) and cerebral metabolic rate of oxygen (CMRO2) mapping. Magn Reson Med. 2020;83:844-857.

63. Stone AJ, Harston GWJ, Carone D, Okell TW, Kennedy J, Blockley NP. Prospects for investigating brain oxygenation in acute stroke: experience with a non-contrast quantitative BOLD based approach. Hum Brain Mapp. 2019;40:2853-2866.

\section{SUPPORTING INFORMATION}

Additional Supporting Information may be found online in the Supporting Information section.

FIGURE S1 To facilitate imaging of ex vivo carotid arteries custom-made 3D printed holders were used for porcine (A) and human (C) carotid arteries. Final sample set-up prior to MR imaging shows porcine (B) and human (D) carotid arteries positioned in $50 \mathrm{ml}$ Falcon tubes and suspended in PBS using the custom holders. Note that 3D printed holders are composed of a bioplastic (polylactic acid) and appearance varies due to the use of poly lactic acid filaments with different colours - silver coloured filament (A) and (C), black coloured filament (B) and (D)

FIGURE S2 Magnitude image (A) and common carotid ROI definition (B) informed by histology for cadaver sample S01. ROIs were limited to normal appearing arterial tissue in the common carotid. Regions of abnormal smooth muscle cells (C), collagen (D and E), elastin (F), GAG (G) and calcium $(\mathrm{H})$, as identified by histology, were excluded from the final ROI. From H\&E histology regions of cell debris indicative of necrotic core (ci), normal appearing tissue (cii) and the cholesterol crystals (ciii) are evident

FIGURE S3 Magnitude image (A) and common carotid ROI definition (B) informed by histology for cadaver sample S02. ROIs were limited to normal appearing arterial tissue in the common carotid. Regions of abnormal smooth muscle cells $(\mathrm{C})$, collagen (D and F), elastin (E), GAG $(\mathrm{G})$ and calcium $(\mathrm{H})$, as identified by histology, were excluded from the final ROI. From H\&E histology regions of thickened intima (ci and cii) and normal appearing tissue (ciii) are evident. Regions (ci) and (cii) were excluded from the final ROI

FIGURE S4 Magnitude image (A) and common carotid ROI definition (B) informed by histology for cadaver sample S03. ROIs were limited to normal appearing arterial tissue in the common carotid. Regions of abnormal smooth muscle cells (C), collagen (D and E), elastin (F), GAG (G) and calcium $(\mathrm{H})$, as identified by histology, were excluded from the final ROI. From histology, bilateral regions of thickened intima are evident and excluded from the final ROI (yellow arrows) FIGURE S5 Magnitude image (A) and common carotid ROI definition (B) informed by histology for cadaver sample S04. ROIs were limited to normal appearing arterial tissue in the common carotid via histology visualising smooth muscle cells $(\mathrm{C})$, collagen (D and E), elastin (F), GAG $(\mathrm{G})$ and cal$\operatorname{cium}(\mathrm{H})$

FIGURE S6 Magnitude image (A) and common carotid ROI definition (B) informed by histology for cadaver sample S05. ROIs were limited to normal appearing arterial tissue in the common carotid. Regions of abnormal smooth muscle cells (C), collagen (D and E), elastin (F), GAG (G) and calcium $(\mathrm{H})$, as identified by histology, were excluded from the final ROI. From histology, a region of thickened intima is evident and excluded from the final ROI (yellow arrow)

FIGURE S7 L-curves of all samples presented in this study used for choosing iterative Tikhonov regularisation parameter, $\alpha$. The optimal iterative Tikhonov regularisation parameter was determined to be $=0.02$ for this study

FIGURE S8 Susceptibility maps produced for the different porcine carotid tissue models (native, decellularised, collagen degraded and elastin degraded) using different susceptibility calculation approaches (TKD, direct Tikhonov, iterative Tikhonov and MEDI). Maps of $\mathrm{R}_{2}^{*}$ presented on bottom row

FIGURE S9 Visual comparison of QSM pipelines including and excluding high-noise regions (tube mask and noise mask) in a representative human cadaver carotid artery. The common carotid ROI was defined in "normal" appearing tissue between the red dotted lines

FIGURE S10 Boxplots comparing susceptibility measurements extracted from vessels $(n=6)$ of each tissue model for different susceptibility calculation approaches (TKD (A), direct Tikhonov (B), iterative Tikhonov (C) and MEDI (D)). Grey data points represent susceptibility measurements in individual vessels. Susceptibility values for tissue models compared using ANOVA and post-hoc test $(* P<.05, * * P<.01$, $* * * P<.001, * * * * P<.0001)$ 
FIGURE S11 (A-D) Comparison between masking pipelines of susceptibility measures in cadaver common carotid. For all dipole inversion approaches, agreement between masking pipelines was excellent using the following criteria - Poor < 0.4, Fair - 0.41-0.59, Good - 0.60-0.74, Excellent $>0.75$ [Ref 50 main text]. (E-H) Boxplots comparing susceptibility measurements in cadaver common carotid artery with fixed porcine carotid artery. Susceptibility values were compared using ANOVA and no significant difference was seen between groups (TKD $P=.7197$, Tikhonov $P=.27322$, Iterative Tikhonov $P=.050082$ and MEDI $P=.559292)$ Comparison between dipole inversion methods in cadaver tissue showed significant differences between methods (ANOVA, $P<.01$ ). Post-hoc testing showed TKD measured a significantly lower susceptibility compared to iterative Tikhonov $(P<.01)$ and MEDI methods $(P<.001)$, direct Tikhonov measured significantly lower susceptibility than MEDI $(P<.05)$. It is also notable that the variation between vessels is reduced with the application of the iterative methods (i.e. wider spread of measured vessel susceptibility in Figure S11A,B,E \& F compared to Figure S11C,D,G \& $\mathrm{H})$. This is due to the inclusion of noise weighting terms and regularisation employed by the iterative fitting methods

How to cite this article: Stone AJ, Tornifoglio B, Johnston RD, Shmueli K, Kerskens C, Lally C. Quantitative susceptibility mapping of carotid arterial tissue ex vivo: Assessing sensitivity to vessel microstructural composition. Magn Reson Med. 2021;00:1-16. https://doi.org/10.1002/mrm.28893 